Papers in Physics, Vol. 13, ART. 130004 (2021)

www.papersinphysics.org

Received: 1 June 2021, Accepted: 12 July 2021

Edited by: L. F. Morales

Licence: Creative Commons Attribution 4.0

DOI: https://doi.org/10.4279/PIP.130004

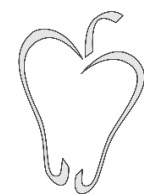

ISSN 1852-4249

\title{
Serendipitous observation of a coronal mass ejection during the total solar eclipse of 14 December 2020
}

\begin{abstract}
G Abramson ${ }^{1 *}$
We report observations of the total solar eclipse of 14 December 2020, during which a coronal mass ejection was seen to propagate. A comprehensive set of photographs covering a high dynamic range of exposure enabled characterization of its dimensions. Displacement of the front can be seen during the few minutes of totality.
\end{abstract}

\section{Introduction}

Coronal mass ejections (CMEs) are violent expulsions of plasma, magnetic field and energy from the Sun [1-3]. During a typical CME millions of tons of solar material are ejected into the heliosphere, within a time scale of about 1 hour. The ejection consists of particles moving at velocities averaging several hundreds of kilometers per second. If suitably oriented, they can reach the Earth after a couple of days, disturbing the magnetosphere and the ionosphere, disrupting radio communications and putting space-borne electronics at risk.

Coronal mass ejections have been recognized as a unique solar phenomenon since the 1970s, particularly after their observation from space with the very sensitive coronagraphs on board Skylab, when they were given a variety of names before the current denomination was settled on [2]. These transient events were soon recognized as being the cause of the high speed solar wind responsible for the geomagnetic storms long observed in radio frequencies, and indeed their effects had been observed on Earth

*abramson@cab.cnea.gov.ar

1 Centro Atómico Bariloche (CNEA), Instituto Balseiro $(\mathrm{UNCu})$ and Consejo Nacional de Investigaciones Científicas y Técnicas, Av. E. Bustillo 9500, R8402AGP Bariloche, Río Negro, Argentina. for thousands of years in the form of polar auroras. After many years of study, and even though it is known that the coronal magnetic field is the driving agent, central questions about their cause and relevance in the dynamics of the corona still remain $[1,3,4]$.

Shortly after their discovery from space-borne observatories, the record of solar observations was searched for possible CMEs, particularly during solar eclipses. Just one instance was found [5]: a drawing made by Gugliemo Tempel during the eclipse of 18 July 1860 in Spain shows a swirl of the size of the solar disk, with a bright core, in the midst of mainly radial streamers of the corona. Several other observers along the path of totality described and sketched a similar structure (but an equal number didn't, indicating the subtlety of the phenomenon to the naked eye); put together, their reports seem to correspond to the development of a CME [5]. Since the times of Tempel, the growing interest in solar phenomena has meant that each eclipse was more observed than the previous ones, yet no other clear observation of a CME was recorded for a full century. Indeed (according to Alexander [2]) the rate of CMEs, their typical duration and the brief periods of totality combine to allow about one chance per century of capturing one during an eclipse. Besides Tempel's remarkable sketch, Filippov et al. [6] mention a little-known 
Papers in Physics, vol. 13, ART. 130004 (2021) / G. Abramson

work showing a sketch (Fig. 18 of [7]) with a structure resembling the front of a light bulb-shaped CME during the eclipse of 21 September 1941, and the seemingly corresponding eruptive prominence (ibid. Fig. 49). We have not been able to find photographs of this eclipse of the era previous to CME recognition; as it occurred during World War II, it was not widely observed. Less clear examples are known, resembling disconnected CMEs in the final stage of evolution [8].

Even though total solar eclipses allow brief observation of coronal phenomena, the tight natural occultation provided by the Moon is ideal for the study of the inner corona, which is normally occulted by coronagraphs below 2-2.5 solar radii ${ }^{1}$, thus hiding about $80 \%$ of the mass of the K-corona and its dynamics [4]. In such a context, both modes of observation of the corona, the continuous one from space-borne coronagraphs and the sporadic one during eclipses, complement each other. Indeed, on a few occasions the phenomena related to CMEs have been observed in this way. Perhaps the most important is the one studied by Koutchny et al. [4,9], who observed the eclipse of 11 August 1999 a few hours before a large CME was observed from space. They were able to identify a possible precursor of the event. They show a large scale structure in the form of a bright arch in the lower corona, with a cavity inside, seemingly rooted in the eruptive prominence that produced the CME a few hours afterwards (see Figs. 10 and 12 in [4]). The event is likely to have been triggered by competing forces due to the the buoyancy of the cavity, on a timescale of hours.

Very recently, Filippov et al. [6] reported the observation of traces of a very slow $(250 \mathrm{~km} / \mathrm{s}) \mathrm{CME}$ during the eclipse of 21 August 2017, using high resolution and high dynamic range photography, with space-based instruments able to follow it before and after. Likewise, during the eclipses of 13 November 2012 and 3 November 2013, Druckmüller et al. [10] were able to observe that the cores of previous CMEs had evolved into tethers connected to tornado-type prominences, providing evidence for the flux of electrons from interplanetary traveling CMEs back to the Sun (see also [11]). Twisted mag-

\footnotetext{
${ }^{1}$ Though some instruments can reach smaller values, like the COR1 instrument on board the STEREO spacecrafts (1.3 solar radii), or the COSMO K-Cor at the Mauna Loa Solar Observatory (down to 1.05 solar radii).
}

netic structures, associated with arches and cavities, were also observed during eclipses in broadband and narrowband images by Habbal et al. [12], highlighting their complex structure and suggesting that their coupling with the cavity material can trigger CMEs. Indeed, these authors recommend dropping the name "cavity" in favor of the more appropriate "hot prominence shrouds".

In summary, total solar eclipses are excellent opportunities to observe the phenomena of the inner corona, complementary to those made with coronagraphs from ground and space. In this work we report observations made during the eclipse of 14 December 2020. Totality touched land only in the Northern Patagonia of Chile and Argentina. As it took place during the travel restrictions imposed by the COVID-19 pandemic, very few expeditions were able to make their way to the path of totality. Coincidentally, a large CME erupted during the eclipse, and its propagating front can be seen in the photographs obtained. From high dynamic range and edge enhanced images we were able to derive its main dimensions and velocity. During the review process of this work, Boe and collaborators presented their own analysis based on images taken by Andreas Möller and Darío Harari [13]. Their observations will be commented on below.

\section{Observational materials and methods}

The eclipse was observed with portable equipment from an outback site near Piedra del Águila, Neuquén province, Argentina. The exact location is latitude $-40^{\circ} 0^{\prime} 18.4^{\prime \prime}$, longitude $-69^{\circ} 59^{\prime} 6.3^{\prime \prime}$, and elevation $450 \mathrm{~m}$; the site lies on the northern shore of Limay river, next to the Pichi Picún Leufú dam. At this site we enjoyed $122.6 \mathrm{~s}$ of totality, between 16:08:03.7 UT and 16:10:06.3 UT (using $\Delta T=69.4 \mathrm{~s}$ and lunar limb correction [14]), at an elevation of $72^{\circ}$. The sky was clear during most of the eclipse, with scattered cumulus clouds at the end of the second partial phase. The wind was very strong, just as had been forecasted, at 50 $\mathrm{km} / \mathrm{h}$ with gale force gusts. The equipment was set up downwind of a very thick bush of Rosa eglanteria, which provided partial shelter under its lee.

The photographic setup consisted of a Canon T3i camera with a Tamron 18-270 mm F/3.5-6.3 Di II 
PApers in Physics, VOl. 13, ART. 130004 (2021) / G. Abramson

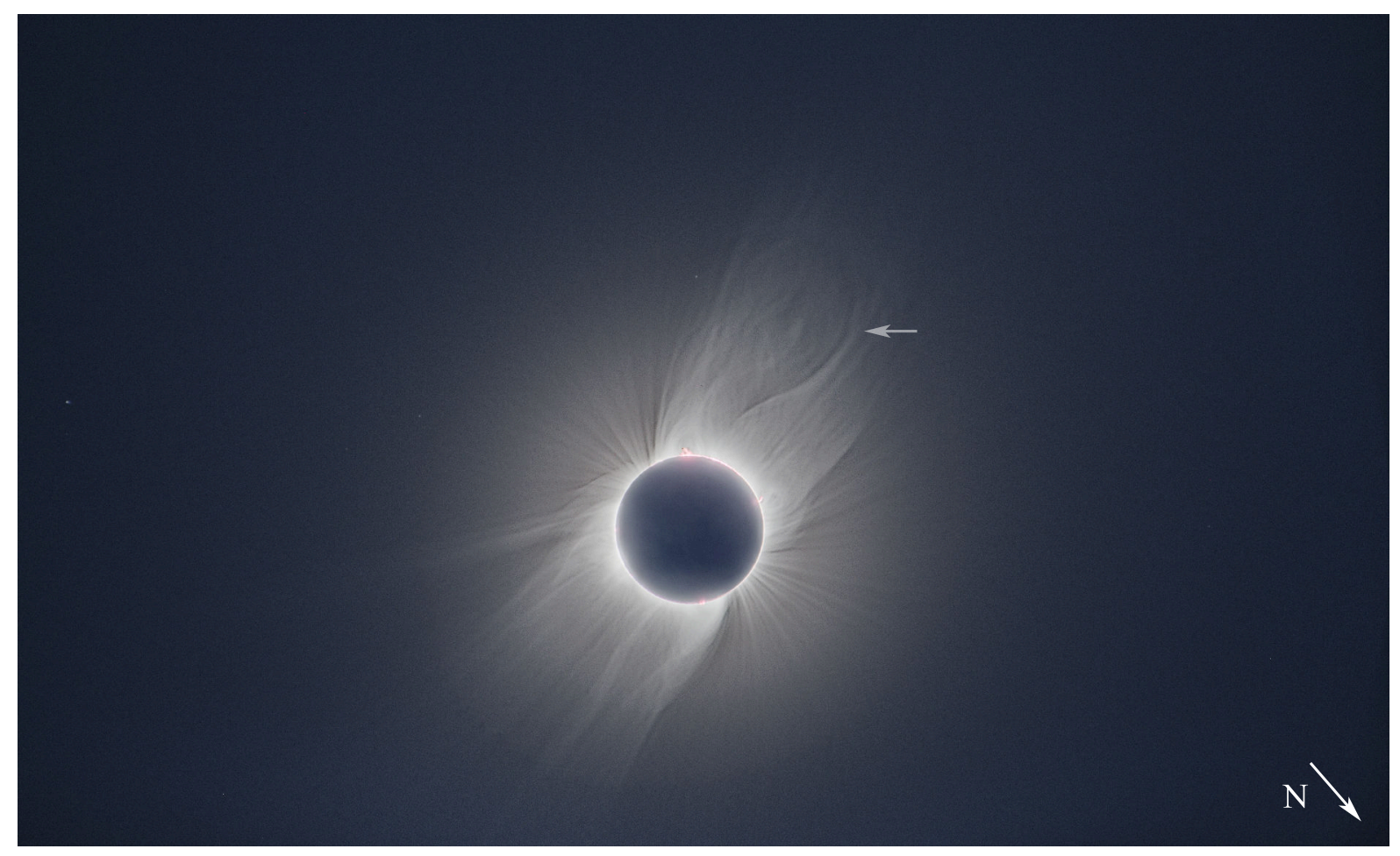

Figure 1: High dynamic range composite corresponding to the first set of images taken during totality (from 16:08:10 to 16:08:21 UT). The teardrop-shaped feature in the corona (arrow) is the CME described in the text. In the full resolution image the comet C/2020 X3 can also be seen (see Supplementary Material - PDF). Solar north is indicated.

VC PZD zoom lens, set at the longest focal length. This provided a field of view of $6.3^{\circ}$ (diagonal), with a resolution of $3.6^{\prime \prime}$ per pixel. The camera was mounted on an iOptron SkyTracker camera mount, which provided sidereal tracking to help keep the Sun in view. Since this is a very simple mount without fine controls, to enable fine adjustments during the eclipse it was mounted, in turn, on a manual equatorial mount and tripod (EQ-1 type). Polar alignment was performed with a magnetic compass, taking into account the magnetic declination of the site. Camera and mount were powered by a $12 \mathrm{~V}$, 17 Ah gel battery.

Exposure control was programmed and run incamera, via a Lua script run by the Magic Lantern alternative operating system available for Canon cameras [15] (see Supplementary Material - LUA). The script ensured autonomous and automatic imaging during all the important phases of the eclipse, including rapid bursts of shooting during totality. Timing was controlled by the camera clock, synchronized in the morning via NTP to an error of less than one second.

During the partial eclipse the lens was covered with a neutral density filter $(N D \approx 5)$, custom made from unbranded polymer film provided by Saracco Astronomía (Buenos Aires). The image was focused manually on the solar limb, using the camera display and its provided magnification. After first contact, the focus was checked using the lunar limb. The filter was removed approximately 15 seconds before totality, and replaced after third contact. The focus was not changed for the unfiltered exposures, since previous trials had deemed this unnecessary.

\section{i Complete set of images}

The automatic capture script was programmed to shoot 7 sets of exposures during the partial phases 
Papers in Physics, Vol. 13, ART. 130004 (2021) / G. Abramson

of the eclipse, each set consisting of a bracket of 3 exposures. Immediately before third contact, 3 relatively long exposures were taken to capture the popular "diamond ring" effect, followed by 7 short exposures in rapid succession to reveal the rugged lunar limb ("Baily's beads"). The same scheme was followed for fourth contact, but in reverse. During totality, the script ran 8 sets of shots ( 7 of which were completed), of varying exposures, each covering a range of 11 photographic stops. At maximum eclipse, a 3-shot bracket of relatively long exposure was taken to capture ashen light and the outer corona. The maximum exposure was 2 seconds, which is about the limit to prevent trailing artifacts with the optical setup implemented. Table 1 summarizes the photographic parameters of the 155 shots obtained.

\section{ii Scale of the photographic images}

For the purpose of taking measurements on the images, we determined a precise scale using the silhouette of the lunar limb during totality. Fifty points were placed at random around the limb, and from their coordinates a least squares fit of a circle produced a radius $r_{m}=275.4 \pm 0.8 \mathrm{px}$ (see Supplementary Material - PDF). We used one of the images with shortest exposure to minimize bleeding of the brightness of the lower corona over the lunar limb (nevertheless, the brightest parts were avoided for the placement of points). Combined with a lunar apparent radius of $1000.45^{\prime \prime}$ (topocentric, derived from DE440 $[16,17])$, we have a scale:

$$
\sigma=3.63 \pm 0.01 \mathrm{arcsec} / \mathrm{px}
$$

\section{Results}

Figure 1 shows a high dynamic range composite of the first set of images, at the beginning of totality. Several large prominences appear around the lunar limb. They could be seen clearly with the naked eye or with small binoculars, contrasting sharply with a very bright, white lower corona. The tear-shaped feature that stands out in the corona is the front of the coronal mass ejection that occurred during the eclipse. The three main components of typical CMEs can be identified: a front, a cavity
Table 1: Complete set of photographs obtained during the eclipse. The eighth set of exposures taken during totality was incomplete at C3. Times of contact (UT) are C1: 14:45:38.4, C2: 16:08:03.7, C3: 16:10:06.3, C4: $17: 35: 52.8$

\begin{tabular}{cccc}
\hline \hline Aperture (F/) & Exposure (s) & ISO & Images \\
\hline \hline Partial: C1-C2, C3-C4 & & \\
11 & $1 / 50$ & 100 & 7,7 \\
11 & $1 / 80$ & 100 & 7,7 \\
11 & $1 / 125$ & 100 & 7,7 \\
\hline \multicolumn{4}{c}{ Second and third contacts: C2, C3 } \\
16 & $1 / 800$ & 100 & 7,7 \\
16 & $1 / 60$ & 400 & 3,3 \\
\hline Totality: C2-C3 & & & \\
8 & $1 / 250$ & 100 & 7 \\
8 & $1 / 250$ & 200 & 7 \\
8 & $1 / 125$ & 200 & 7 \\
8 & $1 / 60$ & 200 & 7 \\
8 & $1 / 30$ & 200 & 7 \\
8 & $1 / 15$ & 200 & 7 \\
8 & $1 / 8$ & 200 & 8 \\
8 & $1 / 4$ & 200 & 8 \\
8 & $1 / 2$ & 200 & 8 \\
8 & 1 & 200 & 8 \\
8 & 1 & 400 & 8 \\
8 & 1 & 800 & 8 \\
\hline Totality (maximum) & $1 / 2$ & 800 & 1 \\
8 & 1 & 800 & 1 \\
8 & 2 & 800 & 1 \\
8 & & & \\
\hline \hline
\end{tabular}

left behind, and a bright core within it. The phenomenon was not visually discernible during totality (at least, it was not noticed by us, but it may have been more apparent to others, as noticed by Eddy [5] regarding the eclipse of 1860); however, it stood out to us immediately when we checked the success of the exposures in the field, after totality (see Supplementary Material - PDF).

The CME originated from active region 12792 , a small bipolar region (area $10 \mu \mathrm{Hem}$ [millionths of a solar hemisphere], Hale class $\beta$, McIntosh class Bxo) which at the time of the event presented four small spots. The region produced a single X-ray flare, GOES class C4.0, which developed between 14:09 UT and 14:56 UT, with a peak at 14:37 UT (8 minutes before first contact at the site of observa- 

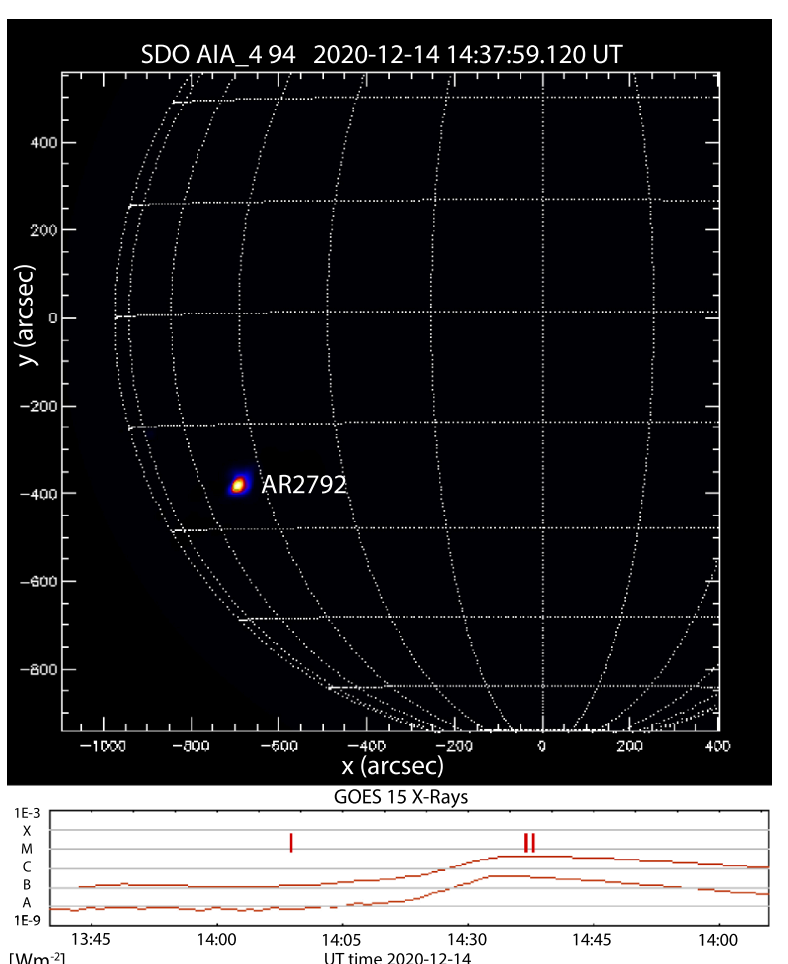

$\left[\mathrm{Wm}^{-2}\right]$

Figure 2: Flare produced by active region 12792 during the eclipse, which produced the CME observed during totality. Top: the position of the flare on the solar disk, from SDO AIA $94 \AA$ images; bottom: the development of the flare as seen in GOES 15 X-ray data. Adapted from SolarMonitor [18].

tion $)^{2}$. Figure 2 shows the location of the eruptive event, identified at heliographic coordinates (S23,E50).

The dimensions of the CME can be determined from our images and solar ephemerides. Figure 3 shows the relevant magnitudes to be determined for the calculation. The angle $y_{1}$ subtended by the longitudinal extension of the CME was measured from the photographs, as was the angle subtended by the transversal dimension, $w$, of the CME front. The angle $y_{2}$ subtended by the solar radius, and the solar distance $r$, were obtained from ephemerides (topocentric at the site of observation, ephemeris

\footnotetext{
${ }^{2}$ From SolarMonitor, hosted by the Solar Physics Group, Trinity College Dublin and the Dublin Institute for Advanced Studies [18]. Data can be accessed at https://www.solarmonitor.org/index.php? region=12792\&date $=20201214$.
}

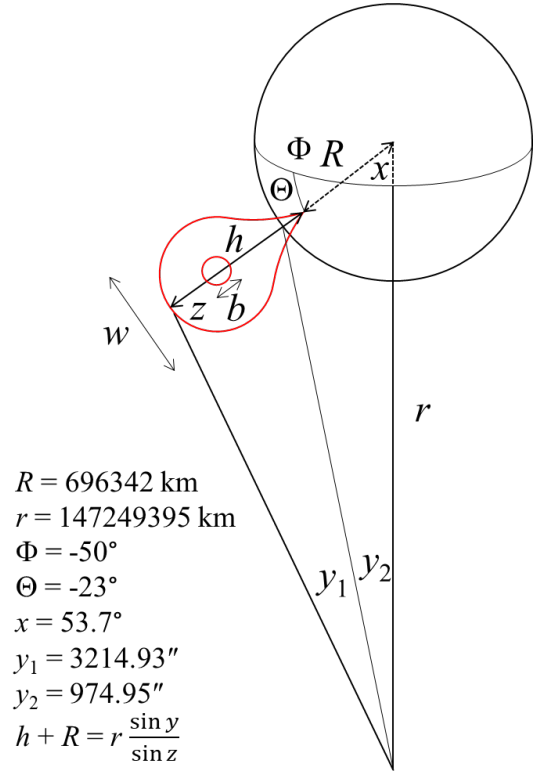

Figure 3: Relevant magnitudes for calculation of the true size of the CME. Parameters $r$ and $y_{2}$ are from ephemeris DE440 [16,17], $R$ is the solar radius [20], and $\Phi$ and $\Theta$ are the heliographic coordinates of the associated flare [18] (from which angle $x$ is calculated). Measured from our images are $y_{1}$ and the angles subtended by $w$ and $b$. Angles $y=y_{1}+y_{2}$ and $x$ give the third angle of the triangle, $z$, and the law of sines enables us to solve the height of the CME, $h$.

DE440 [16, 17]). The heliographic position of the flare enables us to calculate angle $x$, responsible for strong perspective foreshortening of the CME as seen from Earth. Assuming radial development of the CME, these parameters enable us to solve the triangle and calculate the true height of the front of the CME over the solar surface, $h$. A similar calculation (to take into account their closer distance) gives the transverse width of the front, $w$, and the size of the prominent core seen inside the CME, $b$. The main source of error in these derived magnitudes is imprecision in the determination of the position of the front, estimated at $10 \mathrm{px}$ in the image. Combined with the calculated error of the photographic scale, and assuming that the other parameters have negligible errors compared with these, we have calculated an error of $26000 \mathrm{~km}$ for the magnitudes reported in Table 2.

Assuming that the peak time of the flare corre- 
Papers in Physics, Vol. 13, ART. 130004 (2021) / G. Abramson

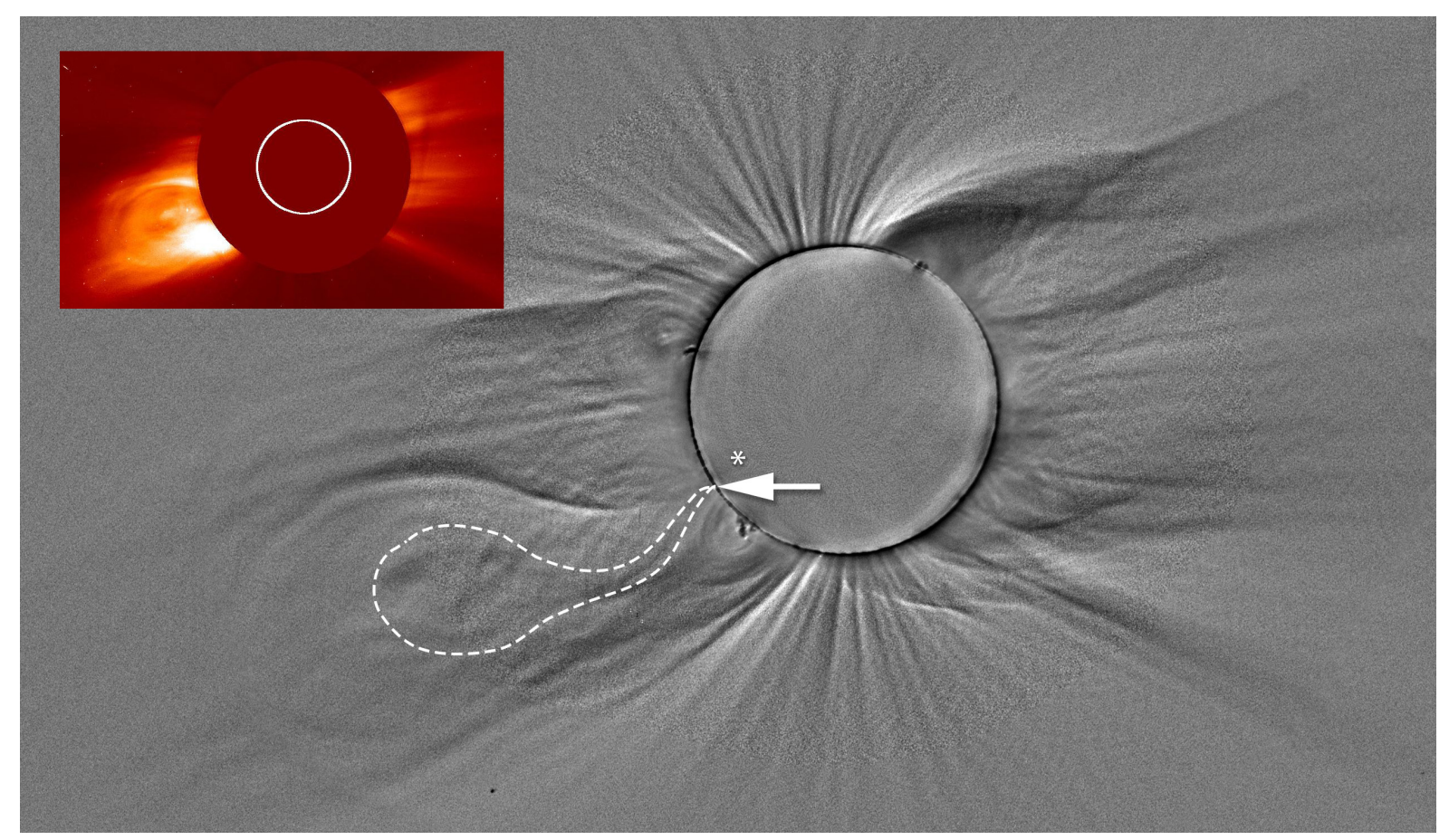

Figure 4: Edge-enhanced view of the solar corona, showing the extension of the CME down to the limb. The asterisk marks the position of the flare. The dashed outline highlights the core and a structure that may connect it to the active region. The inset is SoHO's LASCO C2 image at the closest time (16:12 UT); the red circle is the shadow of the mask of the instrument, and a white circle represents the Sun. Solar north is up. Credit for the inset: ESA/NASA-SoHO/LASCO.

sponds to the start of CME expansion, we know that the CME had expanded for 91 minutes until the time of the exposures. This corresponds to an average radial velocity of $542 \pm 5 \mathrm{~km} / \mathrm{s}$, and a transverse expansion of $108 \pm 5 \mathrm{~km} / \mathrm{s}$ from the middle line. Figure 4 shows another view of the corona, produced by an edge enhancement technique consisting of radially blurring the different

Table 2: CME dimensions, as calculated from photographic measurements. The error is estimated at $0.026 \times 10^{6} \mathrm{~km}$ for them all.

\begin{tabular}{cl}
\hline \hline Dimension & $\times 10^{6} \mathrm{~km}$ \\
\hline$h$ & 2.96 \\
$w$ & 1.05 \\
$b$ & 0.19 \\
\hline \hline
\end{tabular}

exposures and subtracting them from longest to shortest. This view shows that the CME-blown structure still extended to the lower corona, and it was probably still anchored in the chromosphere at the place (marked with an asterisk) where it originated 91 minutes before. The dashed outline shows the bright core and a probable tether connecting it to the active region, in the manner observed in [10]. The inset shows the image at the closest time, taken by the solar coronagraph LASCO C2 [19], on board the Solar \& Heliospheric Observatory (NASA/ESA), showing the corresponding structures in the corona. The image emphasizes the important role of the observation during eclipses, as it enables observation of regions of the solar corona usually masked out by coronagraphs.

The estimated velocity at the distance of the propagating front lies within the limit of resolution of our images for the duration of totality. We made an attempt to measure it directly, with the 
Papers in Physics, vol. 13, ART. 130004 (2021) / G. Abramson

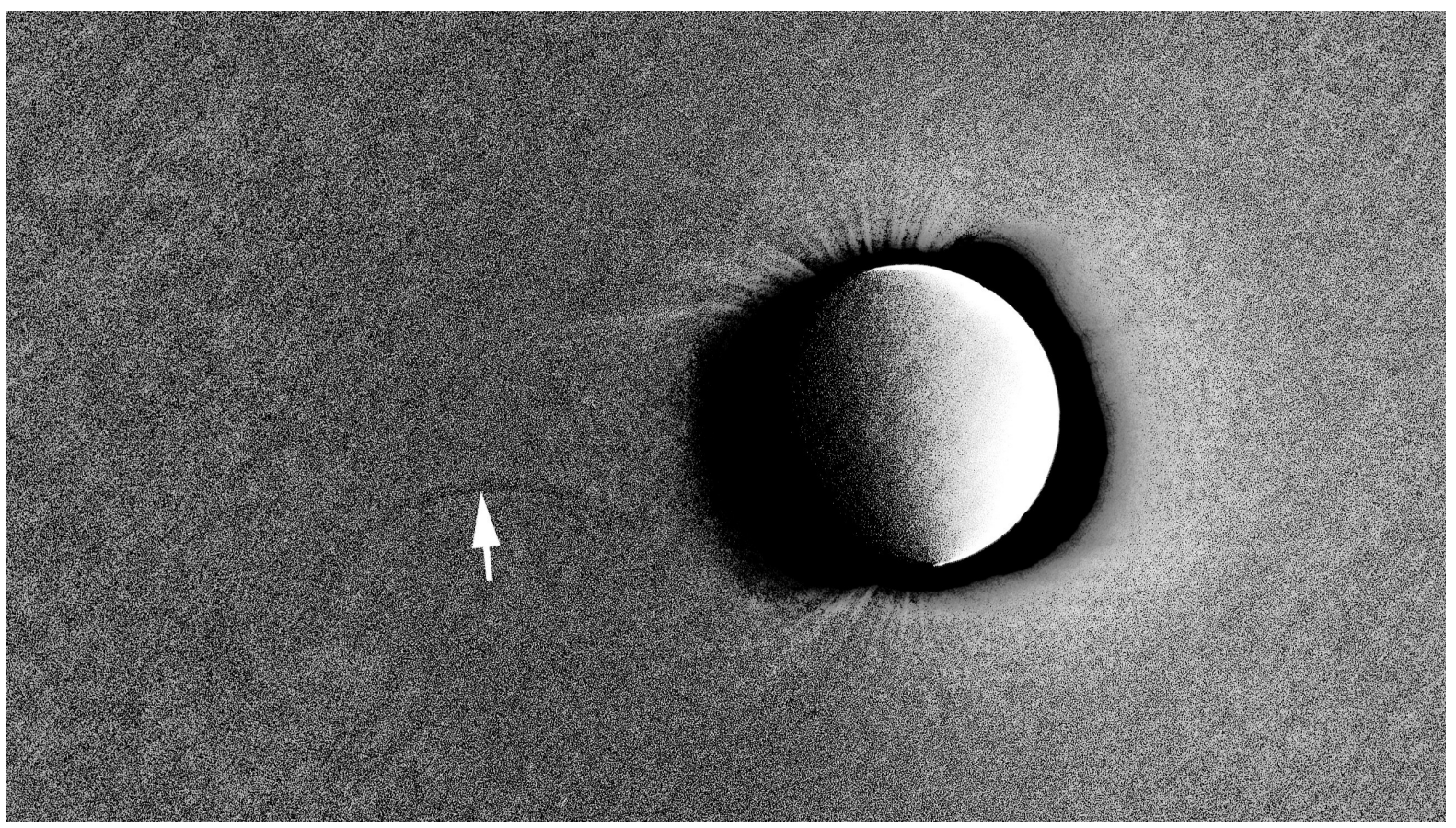

Figure 5: Difference between images taken at 16:09:53 and 16:08:14 (F/8, 1/4 s, ISO 200), unprocessed to prevent distortions. The arrow shows the northern front of the CME, with the greatest displacement. Solar north is up.

result shown in Fig. 5. This shows the difference between two corresponding images of the first and last sets of photographs, unprocessed to prevent the distortions that edge enhancement could introduce. They were registered using two of the stars visible in the field: $51 \mathrm{Oph}$ and $44 \mathrm{Oph}$; the brighter $\theta$ Oph was disregarded for being closer to the edge of the frame where the field is more distorted. An animated version of the two images can be found in the GIF file of the Supplementary Material. The dark stripe signaled with an arrow shows that the northernmost part of the front shifts visibly during the 99-second interval between the two shots. From this feature we have estimated a width of 8-12 pixels, corresponding to an apparent angle of 29-64 arcseconds. At the distance of the core (which is closer than the Sun due to perspective, as estimated before) the displacement corresponds to $18-28 \times 10^{3} \mathrm{~km}$, giving a velocity in the range of $182-283 \mathrm{~km} / \mathrm{s}$, compatible with the transversal expansion rate estimated above. Unfortunately, we were not able to perform a similar calculation for regions closer to the solar surface, since the stars are not clearly visible in the shorter exposures. Other techniques, along the lines of those implemented in the very high resolution images processed by Druckmüller [10], might be able to extract further information from this region.

Indeed, during the review process of this work, Boe, Yamashiro, Druckmüller and Habbal published their own analysis [13], based on two sets of images separated by 13 minutes, taken from two sites along the path of totality. They calculate a velocity in the plane of the sky of $\approx 300 \pm 50 \mathrm{~km} / \mathrm{s}$ for the leading front of the CME. This value is compatible with the transversal expansion determined above. Also, given our estimate of perspective foreshortening, it would correspond to a radial velocity of $\approx 390 \mathrm{~km} / \mathrm{s}$, a value smaller than that calculated above (which is, after all, an average over the previous hour and a half). It is worth mentioning that the authors also characterize this CME as a peculiar structure with two "bubbles." The more apparent is the one discussed here, whereas the other is fainter, with a longer and narrower front which can also be seen in our images, to the south of the 
Papers in Physics, vol. 13, ART. 130004 (2021) / G. Abramson

main one. The authors trace them to the complex interaction of the prominence that erupted and the magnetic field of the active region where the CME originated.

\section{Conclusions}

The serendipitous observation of an ongoing CME, reported here, emphasizes the importance of careful observation of the solar corona during total solar eclipses. This enables us to observe and record details of the corona down to the chromosphere, normally hidden by the occulters of coronagraphs. We have shown that even the displacement of coronal features can be measured during the brief minutes of totality using off-the-shelf photographic equipment. It is our belief that more results could be derived from the set of images obtained, especially in combination with similar records taken to the east of our location, where totality occurred up to 10 minutes later. We hope that a special issue like the present one might bring us into contact.

Acknowledgements - Data supplied by courtesy of SolarMonitor.org and NASA/ESA-SoHO. The author acknowledges partial support from the following grants: CONICET PIP 112-2017-0100008 CO, UNCUYO SIIP 06/C546, ANPCyT PICT2018-01181, as well as the support of Gabriela Casella and María Penovi during the expedition.

[1] R A Howard, A Historical perspective on Coronal Mass Ejections. In: Solar eruptions and energetic particles (Geoph. Monog. Series), Eds. N Gopalswamy, R Mewaldt, J Torsti, v. 165 pag. 7, Am. Geoph. Union, Washington DC (2006).

[2] D Alexander, I G Richardson, T H Zurbuchen, A brief history of CME science. In: Coronal Mass Ejections (Space Sciences Series of ISSI), v. 21 pag. 1, Springer, New York (2006).

[3] T Howard, Coronal Mass Ejections: An introduction, Springer Science, New York (2011).

[4] S Koutchmy, F Baudin, K Bocchialini, J-Y Daniel, J-P Delaboudinière, L Golub, P Lamy,
A Adjabshirizadeh, The August 11th, 1999 CME, Astron. Astrophys. 420, 709 (2004).

[5] J A Eddy, A nineteenth-century coronal transient, Astron. Astrophys. 34, 235 (1974).

[6] B Filippov, S Koutchmy, N Lefaudeux, Solar total eclipse of 21 August 2017: Study of the inner corona dynamical events leading to a CME, Sol. Phys. 295, 24 (2020).

[7] E IA Bugoslavskaia, Struktura solnechnoi korony. In: Trudy Gosudarstvennogo Astronomicheskogo Instituta 19, Izdatelstvo Moskovskogo Universiteta, Moscow (1950).

[8] E W Cliver, Was the eclipse comet of 1893 a disconnected coronal mass ejection?, Sol. Phys. 122, 319 (1989).

[9] S Koutchny, F Baudin, J-P Delaboudinière, A Adjabshirizadeh, The August 11th, 1999 total eclipse CME, In: Proceedings of the Second Solar Cycle and Space Weather Euroconference, 24-29 September 2001, Vico Equense, Italy. Ed.: H Sawaya-Lacoste. ESA SP-477, Noordwijk: ESA Publications Division, p. 55 (2002).

[10] M Druckmüller, S Rifai Habbal, N Alzate, C Emmanouilidis, Tethered prominence-CME systems captured during the 2012 November 13 and 2013 November 3 total solar eclipses, Astrophys. J. Lett. 851, 41 (2017).

[11] S Rifai Habbal, M Druckmüller, H Morgan, A Ding, J Johnson, H Druckmüllerová, A Daw, M B Arndt, M Dietzel, J Saken, Thermodynamics of the solar corona and evolution of the solar magnetic field as inferred from the total solar eclipse observations of 2010 July 11, Astrophys. J. 734, 120 (2011).

[12] S Rifai Habbal, M Druckmüller, H Morgan, I Scholl, V Rušin, A Daw, J Johnson, M Arndt, Total solar eclipse observations of hot prominence shrouds, Astrophys. J. 719, 1362 (2010).

[13] B Boe, B Yamashiro, M Druckmüller, S Habbal, The double-bubble coronal mass ejection of the 2020 December 14 total solar eclipse, Astrophys. J. Lett. 914, 39 (2021). 
Papers in Physics, vol. 13, ART. 130004 (2021) / G. Abramson

[14] X Jubier, Solar eclipses, (2020).

[15] T Hudson et al., Magic Lantern (version lua_fix.2019Mar20.600D102), (2020).

[16] R S Park, W M Folkner, J G Williams, D H Boggs, The JPL planetary and lunar ephemerides DE440 and DE441, Astron. J. 161, 105 (2021).

[17] P Chevalley, Cartes du Ciel (version 4.3), (2021).

[18] P T Gallagher, Y-J Moon, H Wang, Active- region monitoring and flare forecasting - I. Data proccesing and first results, Sol. Phys. 209, 171 (2002). Solar monitor can be accessed at: https://solarmonitor.org.

[19] G E Brueckner, R A Howard, M J Koomen et al., The Large Angle Spectroscopic Coronagraph (LASCO), Sol. Phys. 162, 357 (1995).

[20] M Emilio, J R Kuhn, R I Bush, I F Scholl, Measuring the solar radius from space during the 2003 and 2006 Mercury transits, Astrophys. J. 750, 135 (2012). 